\title{
THE OCCURRENCE OF PASTEURELLA SEPTICA (syn. MULTOCIDA) IN BRONCHIECTASIS
}

\author{
BY \\ R. A. CAWSON AND J. M. TALBOT \\ From the Department of Pathology, King's College Hospital Medical School, and Department of \\ Bacteriology, King's College Hospital, London
}

(RECEIVED FOR PUBLICATION JULY 28, 1954)

There are a number of British reports of the isolation of Past. septica from wounds (Allott, Cruickshank, Cyrlas-Williams, Glass, Meyer, Straker, and Tee, 1944 ; Cooper and Moore, 1945 ; Brunsdon and Mallett, 1953) and from other situations in the human body, such as the appendix (Ludlam, 1944) and joint spaces (Pizey, 1953), but with the exception of the case of sinusitis reported by Bartley and Hunter (1947) the occurrence of this organism in the respiratory tract has not previously been described in this country.

In the following case Past. septica was repeatedly isolated from the sputum of a patient with bronchiectasis over a period of several months.

\section{Case Report}

C. B., a watchmaker, aged 29 , had a history of recurrent attacks of bronchitis and asthma since the age of 10 years. These did not cause him much incapacity until 1950, when he was admitted to hospital with a severe attack of bronchitis; since then he has been cyanosed and has had a decreased exercise tolerance. In February, 1953, he was admitted to the Brook Hospital, London, with purulent bronchitis and finger clubbing, and was suspected of having incipient cor pulmonale. His sputum was examined bacteriologically on March 9, a mixed bacterial flora with no predominant organism being found. $\mathrm{He}$ was discharged from this hospital much improved after six weeks' treatment.

In July, 1953, his condition deteriorated and he was admitted to King's College Hospital dyspnoeic and persistently cyanosed at rest. He was producing a considerable quantity of purulent sputum. A report at this time stated that bronchograms of the right side showed slight dilatation of the upper lobe branches with mild fusiform bronchiectasis, and diminution in volume of the middle lobe with slight dilatation of the dorsal mid-basic and cardiac branches of the lower lobe. On the left side fusiform dilatation of a moderate degree affecting the lingular. middle, and posterior basic segments of the lower lobe was seen. The results of physical examination. of electro- cardiograms, and of cardiac catheterization were compatible with a diagnosis of right heart cardiac hypertrophy due to pulmonary hypertension.

Cultures of his sputum on July 3 and July 10 produced a heavy growth of Past. septica.

A course of aureomycin $(2 \mathrm{~g}$. daily for six days) was given, at the end of which Past. septica was no longer recovered from the sputum, which yielded a heavy growth of Proteus vulgaris; with breathing exercises and postural drainage the patient's condition further improved, and he was discharged on July 22 after three weeks in hospital.

Sputum obtained one month after discharge grew only Proteus vulgaris, but Past. septica was isolated on October 10 from a specimen which yielded a predominant growth of Proteus vulgaris. Sputum examined on November 9 yielded a predominant growth of Past. septica; Proteus vulgaris was not isolated. Though at this time the patient's general condition had deteriorated considerably, there was no reason to believe this was attributable to infection with Past. septica.

As it is suggested that animals are the source of infection with this organism in man, it should be recorded that, since disposing of his dog in 1949, the patient had had no close contact with animals.

\section{Bacteriology}

In smears from the initial aerobic culture the pasteurella was seen as a small Gram-negative coccobacillus measuring approximately $1.5 \mu \times 0.5 \mu$. Bipolar staining was not observed. In cultures on $5 \%$ horse-blood agar plates capsules were readily demonstrated. There was no observed motility either at $37^{\circ} \mathrm{C}$. or at $22^{\circ} \mathrm{C}$.

Cultural Characteristics.-On horse blood agar after 18 hours' aerobic incubation at $37^{\circ} \mathrm{C}$. there was a profuse growth of round, grey, convex, translucent shiny colonies, 1-2 mm. in diameter. There was no haemolysis.

Growth in nutrient broth produced a uniform turbidity and a slight powdery deposit after 18 hours. A gelatin stab showed a filiform growth but no liquefaction after a week. There was no growth on MacConkey's agar after a week. 
Biochemical Reactions.-Acid, but no gas, was produced in glucose, sucrose, mannitol, galactose, sorbitol, and xylose, and weakly in maltose. There was no fermentation in adonitol, arabinose, dulcitol, glycerol, glycogen, inositol, inulin, lactose, raffinose, rhamnose, or trehalose.

The other biochemical tests were as follows:

Litmus milk: no change. Indole: positive.

Methylene blue reduc- Catalase: strongly position: positive.

Methyl red: negative.

Voges Proskauer: negative.

$\mathrm{H}_{2} \mathrm{~S}$ production: negative. tive.

Nitratase: strongly positive.

Citrate utilization: negative.

Pathogenicity Tests.-A guinea-pig inoculated intraperitoneally with $0.5 \mathrm{ml}$. of an 18-hour broth culture of the organism died within 18 hours. One pair of albino mice received $0.2 \mathrm{ml}$. of the undiluted culture intraperitoneally, and another pair $0.2 \mathrm{ml}$. of a 1 in 100 dilution. After 18 hours, one of the first pairs of mice was dead and the other moribund; of the second pair, one mouse was moribund and the other obviously sick.

At necropsy, large numbers of short Gram-negative rods showing bipolar staining were seen in smears of the spleen and heart blood of all five animals.

\section{Serology}

This strain was not agglutinated by any of three “ $O$ " sera (D/5107/51, D/3607/51, D/4307/51) with one of which the majority of animal pasteurellae are agglutinated (Smith, 1953).

A slide agglutination test with the patient's own serum and organism was weakly positive, but tube tests conducted at $37^{\circ} \mathrm{C}$. for somatic antigens and $4^{\circ} \mathrm{C}$. for capsular antigens showed no agglutination above a titre of 1 in 10 .

\section{Sensitivity to Antibiotics}

Using a plate technique with a filter paper strip, the organism was found to be sensitive to penicillin, streptomycin, chloramphenicol, aureomycin, and terramycin.

\section{Discussion}

Although not reported in this country before 1944, human infection by Past. septica is by no means uncommon. The majority of recorded cases have been reviewed by Lévy-Bruhl (1938), Regamey (1939), and Schipper (1947), and fall into two main categories: sepsis following trauma and infections of the respiratory tract. There are a few cases falling into neither class, in which the organism has been recovered from the gastrointestinal tract, from the meninges, from the pericardial sac, and from infected joints. There is almost invariably a history of direct animal contact in cases of wound infection, the majority of which follow the bites of cats or dogs, and, very occasionally, those of other animals, e.g., rabbie and panther.

In the more deep-seated infections, on the othe음 hand, evidence of animal contact is generally lack $\frac{\bar{c}}{\bar{c}}$ ing ; for example, it was present in only one of 13 cases reviewed by Schipper. Regarding respira 2 tory tract infection, our knowledge as to direct) or indirect animal contact is very limited, sincs we have no information on this point from the largest group of these cases. These were reported by Needham, in a personal communication to. Bezjak and Mimica (1952), who states only tha he isolated Past. septica from 28 patients " nearl甲 all of whom were suffering from bronchiectasis."

Too much stress, we feel, need not be given t 8 a history of animal contact in chronic cases, be cause of the well-known ubiquity of Past. septica in diseased and healthy animals, such as cats (Schenk, 1938), dogs (Smith, 1953), and rats (Schipper, 1947); thus it would appear that fe people are able to avoid contact with the organism. on some occasion. There is evidence (Smith, 195 that most pasteurellae isolated from human infecr tion following bites can be recognized serologically as belonging to the types commonly found in the animals concerned, but in at least two cases of human infection without history of animal corf tact, e.g., our case and one of pericardial infectio 3 (Harries, 1954), the strain differed serologically from the usual domestic animal types, a finding which suggests that the source of infection in thesecases was different. On the other hand, some modification may have occurred in the strains during residence in the human body.

While there is no doubt as to the pathogenicit of Past. septica in the case of traumatic infections of the soft tissues, which often involve also the underlying bone, and the occasional infections of serous surfaces or the meninges, the assessment of the role of this organism when found by change in the sputum is much more difficult. In the present case, Past. septica was found in the sputuff on four occasions over a period of several months most often as the predominant organism; it wa thus obviously no chance contaminant of the respiratory tract but an integral part of the brof chial flora. Like the majority of the cases described by Needham (1948) and those of Mulder (1938) and Bezjak and Mimica, this finding was associated with bronchiectasis.

Since it is proverbially difficult to assess the role of any given organism in bronchiectasis, it is impossible to determine the part played Past. septica in the pathology of these cases; 
may well be no more than the colonization of an already damaged bronchial tree.

There is little doubt, as Needham suggests, that Past. septica is more common in the respiratory tract than is generally supposed, and his isolation of this organism from 11 cases in the Mayo clinic during 1947 bears out this contention. This may partly be due to the fact that the cultural characteristics of Past. septica are such that it may easily be overlooked, especially if it is not grown initially in pure culture. Brunsdon and Mallett have suggested that the organism would be isolated more often from wounds of animal origin if routine cultures were taken in casualty departments, and confirmation of this point has been provided by the isolation of Past. septica from two wounds of this nature swabbed as part of a survey of staphylococcal finger infections which was undertaken at this hospital from August to November, 1953.

\section{Summary}

A case of bronchiectasis is described in which Past. septica was isolated from the sputum on several occasions over a period of four months.
The source of infection in this and other cases mentioned in the literature and the possible relationship of the organism to the disease is discussed.

We wish to thank Dr. A. C. Cunliffe for much helpful advice and criticism during the preparation of this paper; Dr. R. S. Bruce Pearson and Dr. W. E. Mahon for allowing us to refer to their patient ; and Dr. K. J. Gurling and Dr. F. Rackow for assistance in preparing the clinical section of this paper. We also wish to thank Mr. J. E. Smith for his interest and for the gift of diagnostic sera.

\section{REFERENCES}

Allott, E. N., Cruickshank, R., Cyrlas-Williams, R., Glass, V., Meyer, I., H., Straker, E. A., and Tee, G. (1944). J.' Path. Bact., $56,411$.

Bartley, E. O., and Hunter, K. (1947). Lancet, 1, 908.

Bezjak, V., and Mimica, M. (1952). Brit. med. J., 2, 757.

Brunsdon, D. F. V., and Mallett, B. L. (1953). Ibid., 2, 607.

Cooper, T. V., and Moore, B. (1945). Lancet, 1, 753.

Harries, E. J.' (1954). Personal communication.

Lévy-Bruhi, M. (1938). Ann. Méd., 44, 406.

Ludlam, G. B. (1944). J. Path. Bact., 56, 307.

Mulder, J. (1938). Acta med. scand., 97, 165.

Needham, G. M. (1948). Proc. Mayo Clinic, 23, 361.

Pizey, N. C. D. (1953). Lancet, 2, 324.

Regamey, $\mathbf{R}$. (1939). Les infections humaines à $B$. bipolari, septicus (pasteurelloses). Huber, Berne.

Schenk, H. (1938). Thesis for doctorate, Munich (quoted by Allin A. E. (1942), Canad. med. Ass. J., 46, 48).

Schipper, G. J. (1947). Bull. Johns Hopk. Hosp., 81, 333.

Smith, J. E. (1953). Personal communication. 